\title{
Hipoglicemia en pediatría: la importancia de entenderla y hacer un buen diagnóstico
}

\author{
Hypoglycemia in pediatrics: the importance of understand it \\ and make a good diagnosis
}

La identificación, investigación y manejo de la hipoglicemia en la infancia y el periodo perinatal continúan siendo una emergencia clínica debido a que la falla en la identificación oportuna puede conducir a un daño neurológico e incluso la muerte. Los avances en biología molecular y las técnicas diagnósticas han ayudado a comprender los mecanismos implicados en la homeostasis del metabolismo de la glucosa y, de esta manera, tener una mejor aproximación clínica del paciente pediátrico con hipoglicemia.

En este número de la revista MEDICINA \& LABORATORIO se presenta una revisión sobre el enfoque diagnóstico de la hipoglicemia en pediatría como paso inicial de la educación médica continua para actualizar al lector sobre los avances en la identificación y caracterización adecuada de las causas subyacentes. No obstante, establecer las causas de hipoglicemia es el punto de inicio necesario para definir y diseñar un plan de manejo apropiado que prevenga las complicaciones secundarias al daño neuronal por el bajo aporte de glucosa.

Es importante tener presente que la evaluación y el manejo de la hipoglicemia en pediatría presenta particularidades propias. En niños, la hipoglicemia persistente casi siempre se debe a defectos congénitos o adquiridos en la regulación de la secreción de insulina, deficiencia del cortisol o la hormona de crecimiento o defectos en el metabolismo de la glucosa, el glucógeno y los ácidos grasos. En las primeras 48 horas de vida distinguir recién nacidos con hipoglicemia persistente de aquellos con hipoglicemia transicional puede ser difícil. De igual forma, los primeros meses de vida representan el periodo más vulnerable para el desarrollo de discapacidad cognitiva. Estas particularidades requieren ser precisadas y tenidas en cuenta al momento del diagnóstico para ser lo más oportunos posible y disminuir el impacto permanente que generan los bajos niveles de glucosa en el tejido cerebral en desarrollo.

En ocasiones puede ser difícil reconocer la hipoglicemia debido a que los signos y síntomas no son específicos y, en algunos casos, un solo valor de glicemia plasmática alterado puede ser un artefacto. Aunque los glucómetros proveen un método de tamizaje que es conveniente para detectar hipoglicemia, su precisión está limitada aproximadamente a $10 \mathrm{mg} / \mathrm{dL}$ a $15 \mathrm{mg} / \mathrm{dL}$ con relación a la glucosa plasmática. Por lo tanto, antes de establecer un diagnóstico de hipoglicemia en pediatría es esencial confirmarla por un método de laboratorio. 
Para los neonatos, los lactantes y los niños pequeños, en quienes la triada de Wipple no se puede utilizar, se requiere la confirmación de la hipoglicemia cuando los niveles de glucosa estén por debajo del rango en el que usualmente ocurren los síntomas neurológicos. Sin embargo, el nivel crítico que determina una intervención en el paciente asintomático continua siendo polémico, al igual que la selección de los lactantes para tamizaje y la frecuencia del mismo.

En el recién nacido persisten muchos retos y preguntas sin respuesta alrededor del manejo de la glicemia durante las primeras 48 horas; sólo el tiempo y los estudios clínicos aleatorizados, controlados y bien diseñados podrán darnos más respuestas. Por lo pronto, siempre será necesario excluir cuidadosamente una enfermedad causante de hipoglicemia persistente en neonatos de alto riesgo antes del alta para asegurar el reconocimiento y facilitar el tratamiento. La terapia a largo plazo se debe basar en la etiología específica del desorden, en conjunto con las recomendaciones del endocrinólogo pediatra y bajo consideración de las preferencias del paciente y la familia.

En el caso del hiperinsulinismo el objetivo es prevenir la recurrencia de las hipoglicemias, que suponen un alto riesgo de desarrollar futuras hipoglicemias no detectables por el fenómeno de desensibilización de la respuesta neurológica a los bajos niveles de glicemia (falla autonómica asociada a hipoglicemia). Para los defectos en el metabolismo del glucógeno y gluconeogénesis mantener la concentración de glucosa sanguínea en el rango normal previene la acidosis metabólica y la falla del crecimiento y, posiblemente, el desarrollo de complicaciones a largo plazo.

Se recuerda al lector que el papel del laboratorio es muy importante en la investigación diagnostica de la hipoglicemia, siendo una pieza que debe articularse con un adecuado raciocinio por parte del equipo médico interdisciplinario tratante. Esperamos que la revisión publicada en este número de MEDICINA \& LABORATORIO logre el objetivo de mejorar el abordaje etiológico de la hipoglicemia y conducir a establecer buenas decisiones de manejo y tratamiento adecuado.

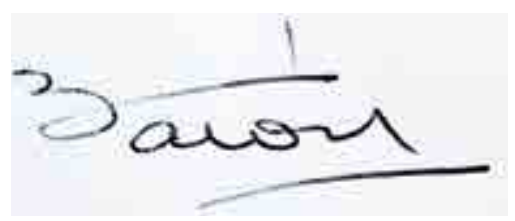

Francisco Barón Torres, MD, Pediatra.

Residente de Endocrinología Pediátrica

Programa de Endocrinología Pediátrica, Universidad de Antioquia

Medellín, Colombia, agosto 2016 